\title{
Ueber die Hitzegerinnung von flüssigen und festen organischen Kolloiden.
}

\author{
Von G. Buglia.
}

(Aus dem Institut für experimentelle Physiologie der K. Universität Neapel, unter Leitung von Prof. Fil. Bottazzi.)

Aus einigen meiner früherenUntersuchungen ${ }^{1}$ ) hat sich klar ergeben, daB die Erscheinungen der Verkürzung des Muskelpräparates durch Wärme irreversibel sind und von den beiden veränderlichen Größen "Temperatur " und "Zeit" abhängen, ähnlich wie die Erscheinungen der Wärmegerinnung einiger organischer kolloider Flüssigkeiten (Blutserum, Eiereiweib usw.). Ferner ergab sich aus anderen Untersuchungen, die ich später anstellte ${ }^{2}$, daß während der thermischen Verkürzungen des Muskels ein Gewichtsverlust eintritt, der durch Erscheinungen von Wasserentziehung $z \mathfrak{u}$ erklären ist, die wahrscheinlich denen ähnlich sind, die, wie man allgemein annimmt, während der Wärmegerinnung der organischen kolloiden Flüssigkeiten eintreten.

Diese Resultate bewogen mich hauptsächlich, die Erscheinungen der thermischen Verkürzung des Muskelpräparates als von der Gerinnung der das letztere bildenden Eiweißstoffe abhängige Erscheinungen zu betrachten und deshalb einen Vergleich zwischen den Erscheinungen der Wärmegerinnung der Eiweißstoffe im Organisationszustande (Muskeleiweibstoffe) und denen der die organischen kolloiden Flüssigkeiten bildenden Eiweißstoffe (Blutserum) anzustellen. Dieser Vergleich zeigte mir bald, daB eine große Aehnlichkeit hinsichtlich der Richtung der die Gerinnungsgeschwindigkeit des Blutserums in Funktion der Temperatur darstellenden Kurve und der Richtung der Kurven der Verkürzungsgeschwindigkeit (Gerinnung) des Muskel- und Sehnengewebes in Funktion der Temperatur besteht.

Um jedoch den Vergleich zwischen den Erscheinungen der Wärmegerinnung der kolloiden Eiweißstoffe im allgemeinen vollständiger durchzuführen, wollte ich ihn vermittelst weiterer Untersuchungen auch auf die Eiweißstoffe im Zustand ihrer größten Reinheit, d. h. wenn sie soweit als möglich der Salze beraubt sind, ausdehnen. $\mathrm{Zu}$ diesem $Z$ weck machte ich einige Bestimmungen der Gerinnungsfähigkeit des Serumalbumins, das, weil ich es einer ver-

1) Archiv. di Fisiol. Volume Giubilare in onore del Prof. G. Fan o, 1909.

7) Archiv. Internat. de Physiol. 1909 (im Druck). längerten Dialyse unterzogen hatte, fast den Zustand vollkommener Neutralität erreicht hatte ${ }^{3}$ ). In der vorliegenden Mitteilung berichte ich über die erhaltenen Resultate, indem ich gleichzeitig auch einige Resultate der früheren Untersuchungen über das Blutserum und das Muskelgewebe anführe, damit man sie leichter miteinander vergleichen kann.

Die in den folgenden zusammenfassenden Tabellen vereinigten experimentellen Resultate sind in einem einzigen Diagramm wiedergegeben, in welchem die Gerinnungsgeschwindigkeit der verschiedenen kolloiden Eiweißstoffe in Funktion der Temperatur darstellenden Kurven (Blutserum Kurve $a$, neutrales Albumin $b$ und $b^{\prime}$, Muskelgewebe $c$ ) nach demselben System von Ordinaten und Koordinaten angeordnet wurden.

Versuche mit Blutserum (Ochse) ${ }^{+}$).

\begin{tabular}{c|c|c}
\hline Versuch & $\begin{array}{c}\text { Temperatur } \\
0 \mathrm{C}\end{array}$ & $\begin{array}{c}\text { Gerinnungszeit } \\
\text { in Minuten }\end{array}$ \\
\hline 1 & 73,2 & $8^{\prime}$ \\
2 & 71,6 & $8^{\prime} 55^{\prime \prime}$ \\
3 & 70,4 & $13^{\prime} 15^{\prime \prime}$ \\
4 & 68,8 & $22^{\prime} 30^{\prime \prime}$ \\
5 & 66,3 & $47^{\prime}$ \\
6 & 63,5 & $123^{\prime}$
\end{tabular}

Versuche mit Muskel (Frosch Gastrocnemius) ${ }^{5}$ ).

\begin{tabular}{c|c|c}
\hline Versuch & $\begin{array}{c}\text { Temperatur } \\
\text { o } \mathrm{C}\end{array}$ & $\begin{array}{c}\text { Verkïrzungszeit } \\
\text { des Muskelgewebes } \\
\text { in Minuten }\end{array}$ \\
\hline 1 & 48 & $0^{\prime} 15^{\prime \prime}$ \\
2 & 45 & $0^{\prime} 30^{\prime \prime}$ \\
3 & 43,2 & $8^{\prime}$ \\
4 & 42,2 & $15^{\prime}$ \\
5 & 41,2 & $33^{\prime}$ \\
6 & 39,5 & $82^{\prime}$ \\
7 & 37,5 & $190^{\prime}$
\end{tabular}

8) Dasselbe neutrale Serumalbumin wurde gleichzeitig bei anderen Untersuchungen ( $\mathrm{B}$ uglia und Karczag, Rend. R. Accad. dei Lincei 1909, im Druck) verwendet, auf die ich verweise, was die Kenntnis der Merkmale betrifft, die es zeigte, sowie die Kenntnis der bei den Bestimmungen der Gerinnungsfähigkeit befolgten experimentellen Methode.

4) Vgl. L. Sabbatani e G. Buglia, Archiv. di Fisiol. 3, 154 (1905).

5) Vgl. G. Buglia, Archiv. di Fisiol. Volume Giubilare in onore del Prof. G. Fan o, 1909. 
Versuche mit "neutralem" Albumin.

\begin{tabular}{c|c|c}
\hline Versuch & $\begin{array}{c}\text { Temperatur } \\
\text { 0 C }\end{array}$ & $\begin{array}{c}\text { Gerinnungszeit } \\
\text { in Minuten }\end{array}$ \\
\hline 1 & 59 & $2^{\prime} 30^{\prime \prime}$ \\
2 & 57,5 & $2^{\prime} 30^{\prime \prime}$ \\
3 & 56 & $3^{\prime}$ \\
4 & 54 & $8^{\prime}$ \\
5 & 52 & $15^{\prime}$ \\
6 & 51,5 & $20^{\prime}$ \\
7 & 50,5 & $65^{\prime}$
\end{tabular}

Versuche mit "neutralem" (konzentriertem) Albumin ${ }^{6}$ ).

\begin{tabular}{c|c|c}
\hline Versuch & $\begin{array}{c}\text { Temperatur } \\
{ }^{0} \mathrm{C}\end{array}$ & $\begin{array}{c}\text { Gerinnungszeit } \\
\text { in Minuten }\end{array}$ \\
\hline \hline 1 & 53 & $1^{\prime} 30^{\prime \prime}$ \\
2 & 50 & $5^{\prime}$ \\
3 & 48,5 & $10^{\prime}$ \\
4 & 47,5 & $18^{\prime}$ \\
5 & 46 & $70^{\prime}$
\end{tabular}

Bei einem Blick auf das Gesamtbild aller Kurven bemerkt man ohne Mühe, daB die Analogie, welche zwischen der Richtung der die Geschwindigkeit der Wärmegerinnung des Blutserums in Funktion der Temperatur $(a)$ darstellenden Kurve und der des Muskelgewebes $(c)$ existiert, auch zwischen diesen Kurven und denen des neutralen Albumins $\left(b, b^{\prime}\right)$ existiert. Diese Analogie in der allgemeinen Richtung der Kurven läbt uns annehmen, daß die Veränderungen, welche die Wärme in organisierten oder nicht organisierten kolloiden Eiweißstoffen bewirkt, stets von derselben Art sind, und zwar sowohl in Abwesenheit als in Anwesenheit der Elektrolyten, die unter physiologischen Bedingungen sich an der Zusammensetzung der kolloiden Eiweißistoffe selbst beteiligen. Dies würde auch durch ein weiteres allen diesen Kurven gemeinsames Merkmal bestätigt, die, wie man sich erinnern wird, in verschiedenen Zeitabschnitten und mittels sehr verschiedener experimenteller Methoden erhalten wurden. Bringt man im Diagramm, indem man die Anordnung der Temperaturen auf den Ordinaten unverändert läßt, auf der Abszisse die Logarithmen der Zahlen an, welche die zur Gerinnung erforderliche Zeit in Minuten darstellen,

b) Fír diese Bestimmungen wurde dasselbe Eiweib, wie fuir die vorigen Bestimmungen, benutzt, nachdem es im Vakuum und auf konzentrierter Schwefelsăure bis auf ein Drittel des Anfangvolums verdampit wurde. so erhält man für jede Kurve eine Linie von fast gerader Richtung. Diese "geraden" Linien sind im Diagramm schraffiert. In der Regel folgt nicht das Segment jeder Kurve, das sich parallel den Ordinaten nach oben hin verlängert und nun oberhalb des Punktes liegt, über den hinaus die Zunahme der Temperatur keine entsprechende Zunahme der Gerinnungsgeschwindigkeit des Eiweißstoffes bewirkt, wahr-

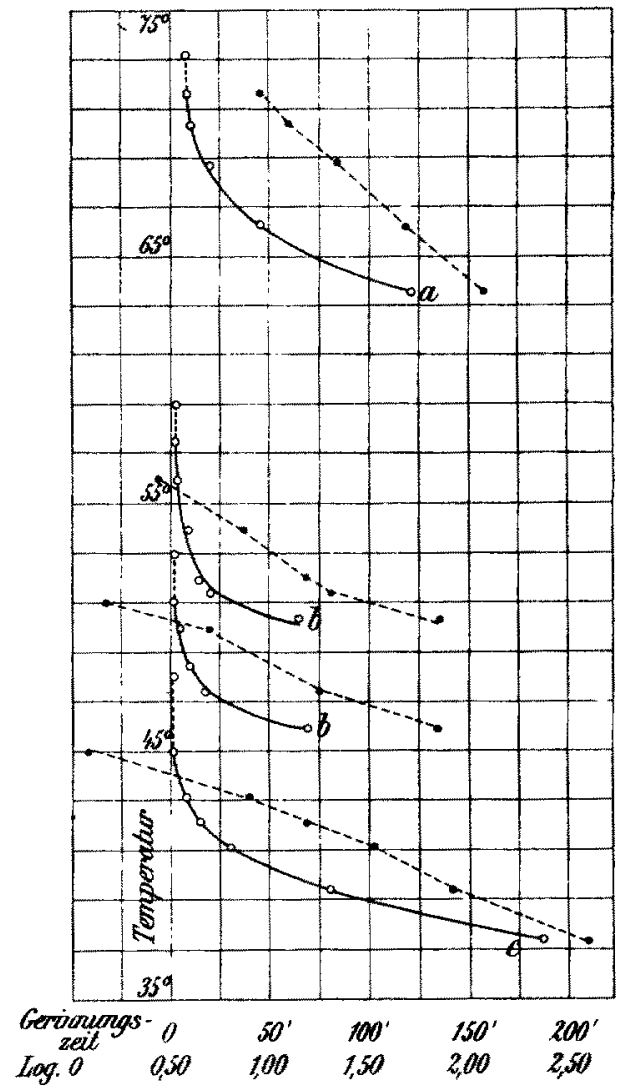

scheinlich weil die Zeitgrenze erreicht ist, die erforderlich ist, damit das Kolloid das Gleichgewicht der Temperatur erlangt. In den Kurven ist dieses Segment punktiert. Beobachtet man jedoch die Kurven, die, wie ich schon sagte, nach einem und demselben System von Ordinaten und Koordinaten gezeichnet wurden, so bemerkt man, daß ihre Lage sehr verschieden ist. Mehr nach unten befindet sich die Kurve des Muskelgewebes, mehr nach oben die des Blutserums und in dem dazwischenliegenden Raume sind die Kurven des neutralen Albumins. Diese verschiedene Lage ist uns ein Zeichen, daf die Leichtigkeit, mit der die verschiedenen kolloiden EiweiBstoffe in der Wärme gerinnen, 
eine verschiedene ist. Betrachtet man nämlich bei jeder Kurve die Temperaturen, die zwischen der maximalen liegen, oberhalb welcher die Gerinnung fast augenblicklich eintritt, und der minimalen, unterhalb welcher die Gerinnung in einer unbegrenzt langen Zeit eintreten würde, so findet man sie viel höher für das Blutserum als für das neutrale Albumin und die Muskeleiweibstoffe. Die Wärmegerinnbarkeit des Blutserums ist also geringer als die des neutralen Albumins und die Gerinnbarkeit der Muskeleiweißstoffe viel größer als die Gerinnbarkeit der beiden vorhin genannten.

Dies alles erklärt sich leicht, wenn man, soweit es die größere Gerinnbarkeit der Muskeleiweißstoffe betrifft, an den besonderen Zustand der Organisation denkt, namentlich aber an die größere Konzentration des Kolloids im Muskel im Vergleich zu seiner Konzentration in organischen Flüssigkeiten. $\mathrm{DaB}$ in der Tat die Konzentration des Kolloids einen beträchtlichen Einfluß auf die Wärmegerinnbarkeit der organischen Kolloide im allgemeinen hat, wird bei meinen Untersuchungen durch die Lage der Kurve $b^{\prime}$ bewiesen, die ich erhielt, als ich dieselbe Lösung von neutralem Albumin, mit der ich die Kurve $b$ erhielt, dem Einflub der
Wärme aussetzte, nachdem ich sie im Vakuum in Anwesenheit von Schwefelsäure so lange konzentriert hatte, bis sie auf ca. $1 / 3$ des Anfangsvolumens reduziert war.

Auch die geringere Gerinnbarkeit des normalen Blutserums im Vergleich zum neutralen Albumin erklärt sich leicht, wenn man bedenkt. dab das neutrale Albumin der Elemente (Hydroxylionen) beraubt ist, die ihm unter physiologischen Bedingungen einen angemessenen Grad von Alkalinität verleihen und, wie bekannt, auf die Wärmegerinnbarkeit der organischen kolloiden Flüssigkeiten eine verzögernde Wirkung ausüben.

Es ergibt sich also klar, dab die Erscheinung der Wärmegerinnung der EiweiBkolloide im allgemeinen stets nach bestimmten Fundamentalgesetzen eintritt, unabhängig von ihrem verschiedenen (flüssigen oder organisierten) $\mathrm{Zu}$ stand, ihrer verschiedenen Konzentration und den eventuellen Schwankungen ihres normalen Salzgehaltes.

Die Leichtigkeit dagegen, mit welcher die kolloiden Eiweißstoffe in der Wärme gerinnen, schwankt merklich je nach den Schwankungen der erwähnten allgemeinen Bedingungen, unter denen sie sich befinden.

\title{
Ueber die biologische Wirkung anorganischer Hydrosole und Salze.
}

\author{
Von Prof. M. Ascoli.
}

(Aus dem Institut für spezielle Pathologie innerer Krankheiten an der k. Universităt Pavia: Prof. M. Ascoli.)

Anlehnend an die Anschauungen $\mathrm{Sch}$ önbein's, der die Zerlegung des Wasserstoffsuperoxyds durch Platinmohr als das Urbild aller Gärung betrachtet hatte, und auf der von Ostwald geschaffenen Grundlage der Lehre von der Katalyse hat G. Bredig die Wirkung der nach seiner Methode hergestellten Metallhydrosole auf das Wasserstoffsuperoxyd in eingehender und mustergültiger Weise in verschiedener Beziehung, insbesondere unter Heranziehung der Methoden der chemischen Kinetik studiert und die mannigfaltigen Berührungspunkte seiner kolloiden Metallösungen mit den organischen Fermenten beleuchtet und mit Nachdruck hervorgehoben; diese Parallelisierung tritt in der Bezeichnung seiner Hydrosole als anorganische Fermente in drastischer Weise zum Ausdruck.

Seit der Betonung solcher Analogien von der Seite Bredig's hat das Studium dieser
Kolloide die Aufmerksamkeit der Biologen in hervorragendem MaBe in Anspruch genommen und eine Reihe von diesbezüglichen Untersuchungen gezeitigt.

Im Mittelpunkte des Interesses stand der kolloide Zustand 'der untersuchten Lösungen mit seiner mächtigen Oberflächenentwickelung und der elektrischen Ladung der Teilchen: er fesselte so ausschlieflich die Aufmerksamkeit der Forscher, daß die merkwürdigen mannigfaltigen biologischen Wirkungen der anorganischen Hydrosole stillschweigend ohne weiteres als Folge ihres physikalisch-chemischen $\mathrm{Zu}$ standes betrachtet wurden, während die übrigen Eigenschaften der verwendeten Metalle so gut wie unberücksichtigt in den Hintergrund traten; ein Standpunkt, der zur Genüge in der Empfehlung verschiedener Kolloide zu therapeutischen Zwecken mit wesentlich identischen Indikationen zum Ausdruck kommt. So erklärt es sich, daß 